\title{
Decreased serum apolipoprotein AII/AI ratio in systemic amyloidosis
}

Toshiyuki Yamada, Tetsuo Ozawa, Fumitake Gejyo, Yasuaki Okuda, Kiyoshi Takasugi, Osamu Hotta, Yoshihisa Itoh

\begin{abstract}
Objective-To investigate if serum apolipoprotein A-I and A-II (apoAI and apoAII) concentrations change in subjects with systemic amyloidosis secondary to underlying disorders.

Methods-Serum concentrations of apoAI and apoAII were measured in 21 multiple myeloma patients, including eight with amyloidosis; 95 rheumatoid arthritis patients, including 45 with amyloidosis; and 73 haemodialysis patients, including 32 with amyloidosis.

Results-ApoAII values tended to be reduced in subjects with amyloidosis in each group, but could not effectively distinguish amyloidosis. However, apoAII/AI ratios were significantly lower in subjects with amyloidosis in all groups. The ratio of 0.2 had diagnostic sensitivity and specificity for amyloidosis; $50 \%$ and $\mathbf{1 0 0 \%}$, respectively, in multiple myeloma; $80 \%$ and $78 \%$, respectively, in rheumatoid arthritis; and $46 \%$ and $90 \%$, respectively, in patients requiring long term haemodialysis.

Conclusion-The apoAII/AI ratio can be a useful biochemical marker of suspect amyloidosis in patients with underlying diseases, especially those with rheumatoid arthritis.

(Ann Rheum Dis 1998;57:249-251)
\end{abstract}

Amyloidosis is a condition in which insoluble fibrillar deposits occupy extracellular spaces and lead to organ dysfunction. ${ }^{1}$ There are several types of amyloidosis in terms of clinical

Table 1 Apolipoprotrein AI and AII concentrations in three patient groups that included subjects with amyloidosis and the diagnostic efficiency for amyloidosis in each group

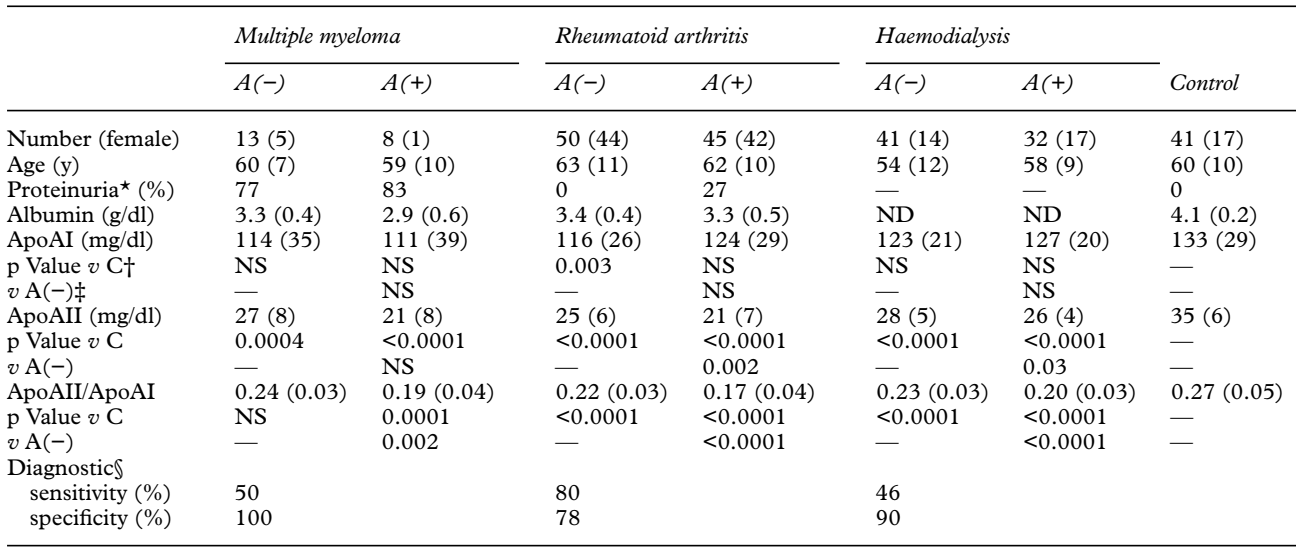

Data are shown as mean (SD). A(-): without amyloidosis, $\mathrm{A}(+)$ : with amyloidosis, NS: not significant, ND: not determined. ${ }^{\star}$ Positive by dipstick test, tversus control, łversus subjects without amyloidosis in each patient group. \$Diagnostic efficiency for amyloidosis in each patient group when the apoAII/AI ratio of 0.2 is adapted as cut off value. features and the protein components that form the amyloid fibrils. Among these are types that are associated with underlying diseases, such as multiple myeloma and rheumatoid arthritis (RA) and in those conditions requiring long term haemodialysis. The involvement of amyloidosis worsens the prognosis of the original disease. ${ }^{2}$ The presence of amyloid deposits can be suspected by symptoms like diarrhoea in RA. ${ }^{3}$ However, some have poor clinical manifestation among patients with amyloid deposits confirmed by histological examination. ${ }^{4}$ As a non-invasive method, scintigraphy, using a labelled serum amyloid $\mathrm{P}$ component, has been proved useful, but not easy to perform. ${ }^{5}$ Therefore, a simple biochemical test would be helpful to detect the presence of amyloid deposits.

Recently, reduction in serum apolipoprotein AII (apoAII) concentrations has been reported in patients with familial amyloidotic polyneuropathy, which is caused by a mutation in the transthyretin gene. ${ }^{6}$ Of importance, subjects with the abnormal gene do not have lowered apoAII concentrations until they have apparent amyloid deposits, indicating that the presence of amyloid deposits may be responsible for the reduction of apoAII values. In this study, we expanded this concept to other types of systemic amyloidosis. The study attempted to determine whether analysis of apoAII can diagnose amyloidosis in the above underlying diseases or in patients undergoing haemodialysis.

\section{Methods} ple myeloma (MM) patients, including eight with amyloidosis; 95 rheumatoid arthritis (RA)
Serum samples were collected from 21 multi-

Fukui Medical School,

Fukui , Japan

F Gejyo

Centre for Rheumatic Diseases, Dohgo Spa

Hospital, Matsuyama,

Japan

Y Okuda

K Takasugi

Department of

Nephrology, Sendai

Shakaihoken Hospital,

Sendai, Japan

O Hotta

Correspondence to:

Dr T Yamada, Department

of Clinical Pathology,

Yakushiji, Minamikawachi,

Tochigi 329-04, Japan.

Accepted for publication 27 February 1998 

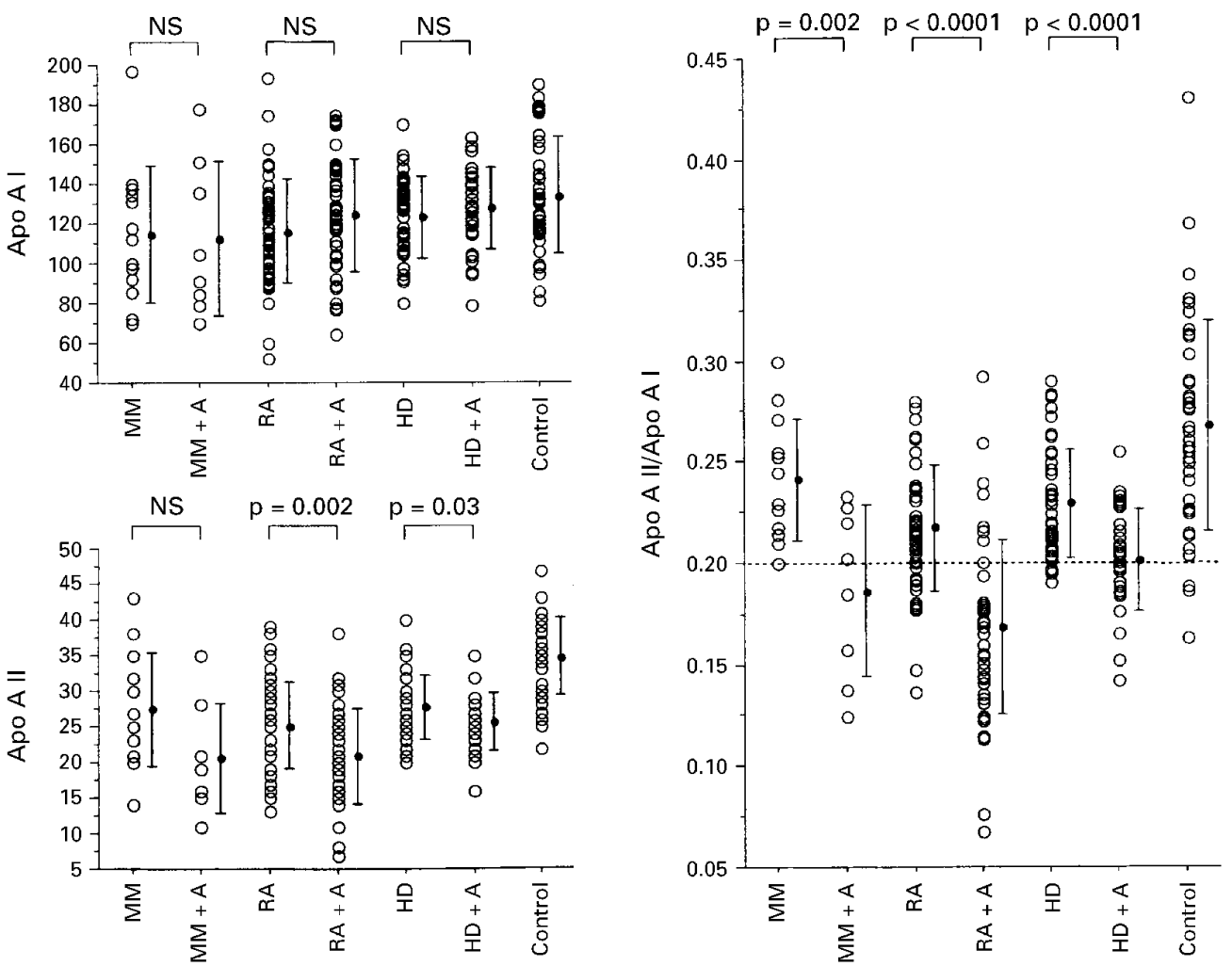

Figure 1 Apolipoprotein AI concentrations (ApoAI) (mg/dl), apolipoprotein AII concentrations (ApoAII) (mg/dl), and the apolipoprotein AII/AI ratio (ApoAII/ApoAI) in patients with multiple myeloma (MM), rheumatoid arthritis (RA), maintainance haemodialysis (HD), including subjects with amyloidosis (indicated by $+A$ ). Bar indicates mean (SD). $p$ Values are also shown also in table 1. NS; not significant.

patients, including 45 with amyloidosis; and 73 patients undergoing maintenance haemodialysis (HD), including 32 with amyloidosis. MM was diagnosed by serum protein analysis and bone marrow cytology. Diagnosis of RA was according to the 1987 revised criteria of the American Rheumatism Association. All the RA subjects underwent histological examination of biopsy specimens of kidney (two cases) or gastroduoedonal mucosa (93 cases), and 50 subjects who were negative for amyloid deposits were defined as RA without amyloidosis. Eight subjects who were defined as MM with amyloidosis were positive for amyloid deposits in rectal mucosa (two cases) or kidney (six cases). Thirteen MM subjects, though not histologically examined, were suspected not to have amyloidosis because of absence of symptoms that would indicate amyloid involvement, such as macroglossia, diarrhoea, or carpal tunnel syndrome. For HD subjects, amyloid involvement was suspected by the presence of carpal tunnel syndrome or cystic changes in radiography of carpal and shoulder bone, which strongly suggest the involvement of amyloid deposits. ${ }^{7}$ As a control, serum samples were also collected from 41 healthy adults. Table 1 summarises the details. None of MM or RA subjects underwent HD. Subjects with familial hypercholesteraemia and cholesterol ester transfer protein deficiency were not included, as these conditions can affect serum concentrations of apolipoprotein independently of amyloid involvement.

Apolipoprotein AI (apoAI), an apoprotein that constitutes high density lipoprotein
(HDL) with apoAII, and apoAII were quantified in serum by an automated turbidimetric immunoassay (Eiken Chemical, Tokyo, Japan). Acute phase serum amyloid A protein (SAA), which can indicate inflammatory activity, were measured by a latex agglutination immunoassay. ${ }^{8}$ Data were analysed using Student's $t$ test.

\section{Results}

ApoAI values were reduced in the patient groups compared with the control group, although not to a statistically significant degree, except in the RA group without amyloidosis. In addition, there was no significant difference in these values between those with and without amyloidosis within each group (fig 1, table 1). In contrast, apoAII values were significantly reduced in all patient groups compared with controls. These values were reduced in subjects with amyloidosis compared with those without amyloidosis in each group and to a statistically significant degree within the RA group (fig 1, table 1). ApoAII/apoAI ratio indicated the involvement of amyloidosis in each group (fig 1, table 1). In the RA group including both amyloidosis and nonamyloidosis, no significant relation between SAA values and the apoAII/apoAI ratio was found (fig 2). The ratio of 0.2 had diagnostic sensitivity and specificity for amyloidosis: $50 \%$ and $100 \%$, respectively, in $\mathrm{MM} ; 80 \%$ and $78 \%$, respectively, in RA; and $46 \%$ and $90 \%$, respectively, in HD (table 1). 


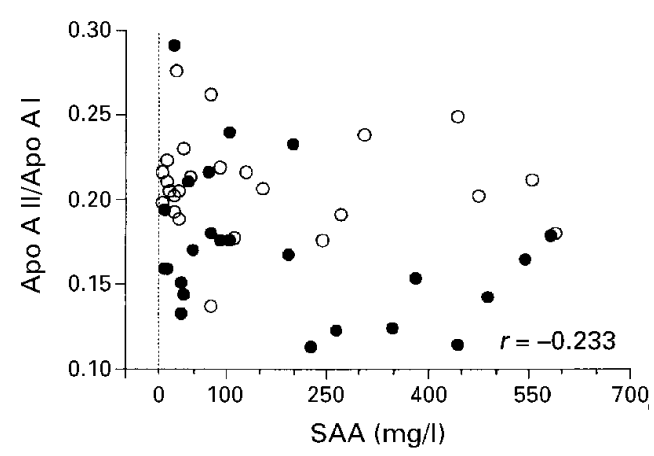

Figure 2 Relation between serum amyloid $A$ concentrations (SAA) and the apolipoprotein AII/AI ratio (ApoAII/ApoAI) in 50 rheumatoid arthritis patients with (closed circle, $n=25$ ) and without (open circle, $n=25$ ) amyloidosis.

\section{Discussion}

Taking the findings of this study and the previous report on familial amyloidotic polyneuropathy together, a reduction in serum apoAII concentrations may be a common phenomenon in systemic amyloidosis. To differentiate amyloid involvement more efficiently, adoption of the apoAII/AI ratio proved successful. This is reasonable, as apoAII concentrations change in good agreement with apoAI, the major protein constituents of HDL. Division of apoAII by apoAI may diminish the effects of quantitative variations in HDL and influential factors common to apoAI and apoAII.

The reason for the reduction in apoAII concentrations in systemic amyloidosis is unknown. Inflammatory conditions may change apolipoprotein composition of HDL, ${ }^{9}$ but the apoAII/apoAI ratio was not affected by inflammatory status, which was indicated by SAA values, in the RA subjects (fig 2). Renal dysfunction and malabsorption should also be taken into account. In the RA amyloidosis subjects, the apoAII/apoAI ratio (mean (SD), 0.16 (0.02)) of 12 non-proteinuric RA amyloidosis subjects were not significantly different from those $(0.17(0.05))$ of 33 non-proteinuric RA amyloidosis subjects. Serum albumin concentrations of each group show no marked nutritional changes in the amyloidosis group (table 1). Thus, the above conditions may not cause a dominant reduction in apoAII, but may reduce both apoAI and apoAII evenly.

ApoAII forms amyloid fibrils in a type of murine senile amyloidosis, ${ }^{10}$ and such mice have low apoAII concentrations. However, apoAII has not been demonstrated in any type of human amyloid deposits. It was shown that the expression of apoAII in the liver was unchanged and secretion of apoAII into urine was increased in familial amyloidotic polyneuropathy, suggesting that HDL from amyloid patients has a lowered affinity for apoAII. ${ }^{11}{ }^{12} \mathrm{It}$ should be investigated whether this is the result of the direct interaction of HDL with amyloid deposits (regardless of type) or the result of changes in lipoprotein metabolism in subjects with generalised amyloidosis.

The apoAII/apoAI ratio was the most effective in RA as a diagnostic marker for amyloidosis. The strict selection of subjects may be responsible for this. Negative results of biopsy do not always rule out the presence of amyloid deposits. A more reliable examination, such as isotope labelled serum amyloid P-component scanning, should be introduced in future studies. The number of MM subjects should be further increased. Poor differentiation of amyloidosis by apoAII/apoAI in HD subjects may be because of the non-biopsy based selection of subjects or may be related to distinct distribution of amyloid deposits, mainly bones and joints, in this type.

In conclusion, the determination of apoAII/ apoAI ratio can potentially be a convenient biochemical method to suspect involvement of amyloid deposits in underlying disorders, especially in RA.

We thank Atsufumi Wada for technical support. This work was supported by a grant in aid for Encouragement of Young Scientists (no 08772184) from the Ministry of Education, Science and Culture of Japan and Clinical Pathology Research Foundation of Japan.

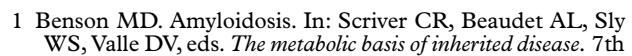
ed. New York: McGraw-Hill, 1995:4159-91

2 Laakso M, Mutru O, Isomaki HA, Koota K. Mortality from amyloidodis and renal diseases in patients with rheumatoid arthritis. Ann Rheum Dis 1986;45:663-7.

3 Okuda Y, Takasugi K, Oyama T, Oyama H, Nanba S, Miyamoto T. Intractable diarrhoea associated with secondary amyloidosis in rheumatoid arthritis. Ann Rheum Dis 1997;

4 Kobayashi H, Tada S, Fuchigami T, Okuda Y, Takasugi K, Matsumoto T, et al. Amyloidosis in rheumatoid arthritis: diagnostic and prognostic value of gastroduodenal biopsy. Br J Rheumatol 1996;35:44-9.

5 Hawkins PN, Myers MJ, Lavender JP, Pepys MB. Diagnostic radionuclide imaging of amyloid: biological targeting by circulating human serum amyloid $\mathrm{P}$ component. Lancet 1988;i:1413-18

6 Ando Y, Tanaka Y, Ueyama H, Sakashita N, Yonehara T, Higuchi $\mathrm{K}$, et al. Low plasma apolipoprotein AII levels in human and mouse amyloidosis with mutant transthretin (Met-30) gene. Ann Neurol 1993;33:101-3.

7 Homma N, Gejyo F, Kobayashi H, Saito H, Sakai S, Suzuki $\mathrm{M}$, et al. Cystic radiolucencies of carpal bones, distal radius and ulna as a marker for dialysis-associated amyloid osteoarthropathy. Nephron 1992;62:6-12.

8 Yamada T, Nomata Y, Sugita O, Okada M. A rapid method for measuring serum amyloid A protein by latex agglutination nephelometric immunoassay. Ann Clin Biochem tion nephelom

9 Coetzee GA, Strachan AF, van der Westhuyzen DR, Hoppe HC, Jeenah MS, de Beer FC. Serum amyloid A-containing human high density lipoprotein 3. Density, size, and apolipoprotein composition. J Biol Chem 1986;261:9644-51.

10 Kunisada T, Higuchi K, Aota S, Takeda T, Yamagishi $H$. Molecular cloning and nucleotide sequence of cDNA for murine senile amyloid protein: nucleotide substitutions found in apolipoprotein A-II cDNA of senescence accelerated mouse (SAM). Nucleic Acids Res 1986;14:5729-40.

11 Tanaka Y, Ando Y, Kumamoto T, Miyazaki A, Nakamura $M$, Nakayama $M$, et al. Changed affinity of apolipoprotein AII to high density lipoprotein (HDL) in patients with familial amyloidotic polyneuropathy (FAP) type I. Biochim Biophys Acta 1994;1225:311-16.

12 Nakamura M, Tanaka Y, Ando Y, Yamashita T, Salvi F, Ferlini A, et al. Decreased affinity of apolipoprotein AII to high-density lipoprotein in patients with transthyretinrelated amyloidosis (Met30, Gln89, Pro36, and Thr34). Biochem Biophys Res Commun 1996;219:316-21. 